\title{
The Observable, the Theory, and Prospective Revised Models for Societal Concerns
}

\begin{abstract}
By Adel Razek
Observational and modeling assessments involve many researchers in fundamental and applied investigations. This article attempts to shed light on these two concepts by underlining their specific uses in different branches of exploration. The nature of the field of research often involves observation, mathematical modeling or both concerns in the form of complementarity. In this work, we discuss for both concepts, the limits of their self-sufficiency. On the other hand, observation comes directly from reality and modeling comes from theory. In this article, we examine the circumstances of model approaches that reflect their intimacy with observed reality. In the case of such a reality corresponding to a societal application, the model often needs a reinforcement to advance towards its objective. A theoretical model generally concerns a given scientific field. In the meantime, the general model governing a societal application involves different theoretical domains. We study how to take into account these areas in the modeling of a given device for a particular environmental behavior. We discuss the choice of such a coupling strategy according to the degree of interdependence of the scientific domains involved. In several constituents of this work, we rely on neuroscience, social science and philosophical concepts. The contribution provides as well a typical application relative to the case of electromagnetic systems that involve magnetic, electrical, mechanical and thermal characteristics. We consider in such complex engineering case examples of different treatments of coupled models, related to different societal applications.
\end{abstract}

Keywords: Observation, Modeling, Societal applications, Revisited models, Coupled modeling, Electromagnetic systems.

\section{Introduction}

This contribution aims to investigate how observables and their models are close to each other and how the two issues of observation and modeling are codependent.

An observable real object behaves according to observable environmental events. We can define the model of such a real object as well as its environment, through the cause and effect of its observed behavior. Such an ascendancy is in generally relative to a science (physical, chemical, etc.) or to a phenomenon involved in a science (electromagnetic, mechanical, etc.).

We can carry on the analysis of a real object and its behavior according to real environmental events by observation and/or modeling. We can have an interrogation on the self-sufficiency of each of these two issues of analysis as well

*Emeritus Research Director, C.N.R.S. \& Honorary Professor, CentraleSupelec, GeePs, University of Paris-Saclay and Sorbonne University, France. 
as on their complementarity. The nature and field of investigation closely related to this questioning have specific considerations. Observation or modeling can be self-sufficient in areas of investigation that we often consider idealized. In the general case of real societal landscapes, we use the two issues of analysis in a framework of complementarity. Therefore, even in the domains calling usually for observation issue, this is in commonly not self-sufficient; (see for example, LéviStrauss 1958).

Also for the domains calling usually for modeling issue, this is in general not self-sufficient; (see for example, Merleau-Ponty 1960). Concerning the disagreement of a real observable and its model, we know that the first is correct (reference) and that it is necessary to revise the model to approach reality. Most of mathematical models originate of coherent and pleasant theories concerning one branch of science under idealizing assumptions concerning the environmental conditions such as temperature, pressure, etc., and the nature of materials such as homogeneity, viscosity, linearity, etc. In the real background of society, the environment and matter rarely meet such ideal conditions. In some special cases, we can take into account these imperfections by revising or adding specific coefficients. In the general case, the imperfections of the scientific branch implicated concern other scientific areas. Moreover, in certain societal panoramas, several branches of science may govern the behavior in question. In all these cases, the revised final model will be a coupled model resulting from the association of different theories of different scientific branches.

We often encounter such real societal applications. For example, we can take electromagnetic systems in general. The nature of the physical phenomena governing electromagnetic systems is complex and a complete model should take into account the magnetic, electrical, mechanical and/or thermal characteristics. The corresponding variables may be interdependent and the parameters may vary due to these variables. A physical model is, more or less coupled because of the importance of the interdependence of its related phenomena.

\section{Observation Auto Sufficiency}

We will consider first the domains of investigations that generally call for observation. A typical domain among these is Anthropology. We will consider an important field of this domain that is Myths. This field has used principally observation since its creation. This was mainly due to the richness of memory that is necessary for deep investigations in this field. In opposition, despite this richness of memory, the observation alone rapidly appeared limiting the extension of the field of research. Putting it in a theoretical context such types of investigations opens this field. Claude Lévi-Strauss (1908-2009) has illustrated this, in "Structural Anthropology", Paris 1958, (see Lévi-Strauss 1958). He elucidated clearly in this work that structural researches in the social sciences are indirect consequences of modern mathematics: logic, sets, groups and topology. These theoretical "tools" make it possible to generalize a more in-depth research involving different studied situations combined in the same model (theory). 
Consequently, these isolated observations may feed into a more general structured concept elucidating real universal phenomena. This example shows clearly that observation alone, even may be auto sufficient in certain situations, needs generally the modelling issue complementarity.

In physical science, we can consider a second example concerning the work of Michael Faraday (1791-1867) in the field of electromagnetic induction. Faraday was an excellent observer who transmitted his ideas resulting of his experimental work in a very simple style. His mathematical skills, however, are limited to the simplest algebra. The contributions of Faraday and others, to put them in a mathematical context and summarize them into a set of equations inspired James Clerk Maxwell (1831-1879). These "Maxwell equations" are the basis of all modern theories of electromagnetic phenomena and are the origin of many scientific research works in this area, as we will show in the last part of the present paper.

This complementarity (modeling helping observation) exists in many other areas of investigation in all fields of science, for example, demography, sociology, neuroscience, physical sciences.

\section{Auto Sufficiency of Modeling}

On the other hand, if we consider the areas of investigation that generally require modeling, for example physical, chemical, etc. In such cases, the establishment of coherent and pleasant theories (models) often requires assumptions simplifying or idealizing the real context of investigation. When the situation studied is very close to such an idealized context, the problem of modeling analysis could be self-sufficient. On the contrary, in most societal survey situations, this type of analysis will not be enough.

The great philosopher Maurice Merleau-Ponty (1908-1961) analyzed and commented on such situations in "The eye and the spirit", Paris 1960, (see Merleau-Ponty 1960). He observed, concerning isolated-use of theory, that science manipulates things, renounces living inside and considers the world as an object of knowledge "dissociated" from the existing subject. In addition, he added with regard to the models, that they are function of their authors, that there is correspondence between the model and the spirit, and that the scientists see the world with a spirit related to the model disregarding the reality observation. Therefore, theory cannot be used isolated of observation.

The Merleau-Ponty reflections suggest the existence of a correlation between the model, its author and the minds of its users. Nearly fifty years later, and thanks to research on modern neuroscience, the theory of mirror neurons has corroborated this suggestion. This work first showed for animals, then for humans, thanks to functional MRI brain mapping, that the zones of activated neuronal connections are similar, involving an observable and one or more observers, (see for example, Ferrari and Rizzolatti 2014).

It is interesting to note that, in general, one can consider a theory only established after validation by observation. Moreover, such a theory remains valid 
until disagreement with observation. This shows the importance of observation in the foundation of science and therefore the theory-observation couple is necessarily always associated and the complementarity (modeling accounting for observation) exists in investigations in all fields of science.

\section{Societal Scenery and Complementarity}

From the analysis and discussion presented, it is obvious that in a typical societal application (real observable) in domains such as mobility, health, or security, we need a complete complementarity of the issues of observation and modeling. In fact, we know that the observable is reality and that the theoretical model is indispensable. When such a model does not correspond to the observable, we must revise it to bring it closer to the observable. One can consider this revision in the model in two different ways, either by approximation or by taking into account more theory in the model. Both approaches can lead to a better match of the observable and the model. In fact, approximation matching is often accidental and a bad option because "the adjustment is not necessarily tighter". Simplified models often correspond to non-universal approximations that incorrectly exercised for every one observable and can become dangerous through "recipes" operated in certain specialties. Therefore, we can only consider a correct revision in the model by taking into account more theory in such model.

\section{Non-Universal Modeling Panoramas}

As mentioned earlier, mathematical models come from coherent and pleasant theories under idealizing assumptions. When such theories apply to situations corresponding to these hypotheses, the model will represent reality; for example, in the case of electromagnetism, the conditions of infinitely small or large.

On the other hand, in the application of a complete theory on a situation in which a part (term) is negligible or non-existent; the corresponding approximate model will represent reality. For example, in the case of electromagnetism, we can neglect eddy currents in the modeling of nonconductive materials.

We cannot generalize the models considered in these two cases to the general cases of societal applications and in any case, we cannot consider them universal.

\section{Revised Model Closer to the Observable}

As mentioned earlier, if we consider the case of a model in an idealized context, the problem of modeling analysis could be self-sufficient. Considering a more realistic observable societal situation, the idealized model will not represent the behavior of the landscape. Only a revised augmented model involving more theory to account for the realistic aspect can represent such a panorama. The idealized context often corresponds to the disregarding of existing phenomena that governed by other branches of science. The augmented model must take into account these additional occurrences. In fact, such a revised model corresponds to 
the processing of the idealized model associated with such surplus occurrences in a sort of mathematical cocktail. Because of this objective, we can consider such a treatment by systematic iterations between the mathematical models concerned by this cocktail. If these mathematical models are particularly interdependent and notably with complex behaviors laws, only a coupled solution of these models can give an image "closer to the observable" of this type of behavior.

\section{Coupled Solution}

The coupling of interdependent models could be weak, strong or intermediate. The degree of this interdependence corresponds directly to the degree of coupling required.

In the case of a weak coupling, one can consider the models individually in an iterative process involving their interdependent behavioral laws. We can call such case a weakly coupled (or a separately iterative coupled).

For the other limit of strong coupling, we must consider a simultaneous solution of mathematical models containing their behavioral laws. We can call this case a strongly coupled (or simultaneously coupled).

For a given degree of coupling the choice of, an appropriate mathematical formulation, suitable space and time scales and a correct resolution method can lead to the right behavior. In the general case, we use three-dimensional discretized geometrical cells or elements for the different space scales and a discretized form for the time. The degrees of discretization refinement of space and time depend on the complexity of the geometry, the nature of temporal evolutions of phenomena, and the individual and interdependent laws of behavior of variables.

\section{Type of Exploration and Area of Expertise Requirements}

\section{Analysis and Reflection Depths}

As stated earlier, we can approximate (idealize) the studied landscape or consider it completely in its real societal form.

In such idealized case, we are in the presence of a simplified model that the use obeys a given situation. In such a case, once we have chosen the model (as a tool), we do not need to think deeply and simply execute a sort of reflex.

In the second real societal case option, we need to consider an appropriate model. In such a case, we must properly examine the behavioral conditions of the scene and decide on the appropriate model respecting these conditions. In this case, we usually need a precise model of the coupled type. Unlike the idealized case (involving a reflex), it is necessary in this case more reflection (cogitation).

The two situations above (idealized and real), call for two completely different modes of thought (reflex and negotiation).

Again, thanks to research on modern neuroscience, the theory of neural 
plasticity clearly illustrates the difference between these two situations. Using functional MRI brain mapping, we can notice, for different situations requiring absolute concentration for a given action without any dispersion (no reflection), that complex multiple neuronal connections become extremely simple thanks to plasticity, (see for example, Adkins et al. 2006, Nielsen and Cohen 2008). We encounter such situations, for example in sports competitions where we need a very high level of concentration to achieve a precise action. We call this state "reflex state". This is the opposite state to that one where reflection maneuvering complex neuronal connections.

In case of deep reflection (cogitation or negotiation), the brain acts as a predictive system (Bayesian brain) operating in situations of uncertainty. Our reflection on the surrounding observables is not, established solely on the information of our perceptions. In its place, what we observe is, as well deeply transformed by the circumstance of our feeling and our prospects about it. The predictive model assumes that our brain generates sensory expectations at every moment and that only the error (difference between prediction and observation) exists in the transmitted neuronal discharges. In many areas of cognitive neuroscience, the Bayesian brain suggests a great capacity for statistical inference at several levels (perception, action, language, etc.); (see for example, Knill and Pouget 2004, Pouget et al. 2013, Amalric and Dehaene 2017).

\section{Specialties of Modeling}

The specialty (business) involving modeling-related activities could belong to different categories, from basic mathematical formulations to simple users of closed black box tools. We can classify these categories into two distinct professions. The first concerns the development and improvement of models with the aim of being as close as possible to the real observable landscapes. The second concerns the use of modeling tools. We can call the first developer and the second user. We can cite the following examples for these two professions: Developing a strong coupling tool (code) considering a simultaneous solution of mathematical models containing their behavioral laws is the occupation of a developer. The use of such a tool by a designer is the occupation of a user.

Developers are usually involved in experienced teams in applied mathematics, numerical analysis, computer science and the scientific fields concerned with the developed tool. It may be noted that, in such activity we meet general evolutions in the fields of, par example, computation capacity, algorithmic optimization, instrumentation, etc. The developers are supposed to work closely with the users.

These users must be experts in the tool specifications and involved in the teams working in the heart of societal sceneries. By definition, a user far from either the tool or the societal domain could be hazardous. It is notable that, rapid technological developments and fields that are more specific generally characterize societal sceneries.

\section{Case of Electromagnetic Systems (Application)}




\section{Introduction}

Electromagnetic systems are present in most of societal applications: mobility, health, security, communication ...These systems behave under four occurrences: electrical, magnetic, mechanical, and thermal. In electromagnetic systems, we need generally to consider the coupling of the magnetic field with the other occurrences. Among the concerned couplings, one is integrated (electrical and magnetic), while the others are causative; (see Piriou and Razek 1993, Ren and Razek 1994, Piriou and Razek 1990, Ren and Razek 1990, Sekkak et al. 1994, Sekkak et al. 1995).

The first three occurrences have relatively rapid time evolutions (small timeconstants). The forth one (thermal) has a slower time evolution (higher timeconstant). Moreover, apart from the classification of integrated and causative occurrences, we have the case of material intrinsic manifestations. This concerns mainly smart materials where the functioning involves two of these occurrences; for example, the case of piezoelectric materials that the functioning calls electrical and mechanical (deformation) behaviors. As well as, the case of magnetostrictive materials, that involve magnetic and mechanical (deformation) in their functioning. It is noteworthy that in electromagnetic smart systems that involve such smart materials, we may have a mixture of occurrences: integrated, causative, and/or material intrinsic.

In the general case, the determination of the system behavior for a given functioning, needs the solution of the equations of the involved governing occurrences. As the system structures are generally of complex geometry and involving materials with non-linear behavior laws, we need the local distribution of variables. Due to this, we use 2D or 3D discretized geometrical cells or elements to obtain the local solution of the needed variables. On the other hand, the nature of the concerned system behavior implies either a frequency domain analysis or a time domain analysis (discretized form of the time). The degrees of discretization refinement of space and time depend on the complexity of the geometry, the nature of temporal evolutions of phenomena, and the individual and interdependent laws of behavior of variables.

In the general case, for structures affected by independent occurrences with very different time constants (very different time evolutions) and involving, a material that behaves linearly, we need a simple weak coupling solution (direct separate solutions). However, if the material behaves non-linearly and/or the involved variables are interdependent, the weak coupling will be separately iterative.

In the contrary, in the case of occurrences with the same order of time constants (near time evolutions) and materials with non-linear behavior; we need a simultaneous strong solution. The non-linearity, as well as the variables interdependence, are included in the simultaneous solution through iterative convergence procedure

Therefore, for devices affected by two or more characteristics (occurrences) of those three with small time-constant, we need generally a strong coupling 
(simultaneously coupled) except the case of linear behavior; (see for example, Piriou and Razek 1993, Ren and Razek 1994). For those concerned by two occurrences including the thermal one, we can use a weak separately iterative coupling; (see for example, Sekkak et al. 1994, Sekkak et al. 1995). We will consider in the following examples these two cases.

\section{Electromagnetic Integrated Interaction}

An electromagnetic system is composed of an electrical circuit and a magnetic circuit. These two circuits are essentially independent in their construction but not in their design. Therefore, we must consider the electrical circuit when exploring electromagnetic systems. A weakly coupled model does not represent reality in the general case. The solution of the problem depends on the laws of behavior and the topologies of these circuits. In the case of linear circuits, weak coupling of electrical and magnetic circuits can be used (Piriou and Razek 1990). In the case of nonlinear magnetic or electrical circuits, only a strongly coupled (simultaneous solution) model can be used (Piriou and Razek 1993). On the other hand, considering the topological aspect, in the analysis of electromagnetic structures simultaneously considering the magnetic and electrical equations (strong coupling), many works have been exposed in the 2D case; (see for example, Ancelle et al. 1980, Nakata and Takahashi 1986, Konrad 1981, Shen et al. 1985, Pawlak and Nehl 1988). Generally, in such a case one solves the magnetic equations by means of a formulation with the magnetic vector potential. The coupling is achieved through the conductor current expressed in terms of current density and the flux linkage expressed from the vector potential. In the case of 3D geometries, different formulations can be used according to the studied problem. The problem could be magnetostatic or magnetodynamic (involving eddy currents). The analysis could be in the frequency domain or the time domain. In Piriou and Razek (1993), the problem of strong coupling of magnetic and electric equations is analyzed in its general form. A 3D (or 2D) model for time domain finite element analysis based on the simultaneous solution of non-linear (or linear) magnetic and electric equations is presented and illustrated by several application concerning different devices.

\section{Magneto Mechanical Causative Interaction}

We consider the case of magneto mechanical interaction. In electromagnetic devices, the magnetic materials are subject to an elastic deformation under the charge of magnetic forces. In reverse, the magnetic field and the force distributions are, more or less influenced by the deformation. The study of such an interaction needs a simultaneous consideration of magnetic and mechanical fields. The degrees of coupling are different according to the significance of the interaction. For example in electrical machines, the deformation generated by magnetic forces is generally small and the variation of the magnetic field distribution is negligible. In such case, we can solve the magnetic and mechanical problems separately (weak coupling); (see for example, Ren and Razek 1990). However, in other 
applications, for example magnetic forming, the deformation (or the displacement) is essential and modify the magnetic field and therefore the magnetic force distribution. This change is due to two facts: The first is the change of mechanical structure. The second is the change of magnetic permeability with mechanical stress (magnetostriction). Such case corresponds to strongly combined magnetic and mechanical phenomena and so we need to use a strong-coupled model (Ren and Razek 1994).

\section{Magneto Thermal Causative Interaction}

Concerning magneto thermal coupling, we can consider a specific medical application relating to hyperthermia techniques in the treatment of cancer (Sekkak et al. 1995). In this case, the thermal behavior is very slow (high time constant) with respect to this of the electromagnetic radiation (very low time constant). In such a case, we can solve separately the two governing equations in an iterative process (weak coupling). Therefore, when studying the potential of such techniques, it is possible to calculate the temperature distribution in biological tissues produced by electromagnetic radiation that is determined separately. With this objective, it is possible to use a three-dimensional thermo-electromagnetic model for the analysis of high-frequency electromagnetic heating of biological bodies. The accumulation of electromagnetic energy is determined using threedimensional local field computations. One can compute the local thermal distribution from the generated thermal power with a three-dimensional model. In these calculations, we can use a dielectric material with losses whose physical parameters are those of biological tissues (such as fat and muscle).

\section{Material Intrinsic Interactions}

As mentioned before, the case of smart materials that their functioning uses properties of the basic four occurrences, needs the consideration of coupling. The study of such an interaction needs a simultaneous consideration. The degree of such coupling depends on the time constant of the phenomena as well as the laws of behavior of the material. In case of material linear behavior and/or very different time constants of phenomena, we can use a weakly coupled solution. Otherwise, we need a strong coupling.

Considering the case of piezoelectric (electrostrictive) materials that present a linear behavior, the solution involves a weak coupling of electrical and mechanical deformation. In such case, the presence of an electric field produces a deformation (direct effect) and the application of a stress induces an electric potential (inverse effect). We can use the benefit of these properties for sensor, actuator or electric source applications.

In reverse, the case of magnetostrictive materials that behaves non-linearly, we need a simultaneous strong coupling. Different applications use magnetostrictive materials that present magnetic and mechanical properties, which are strongly coupled. In such case, the presence of a magnetic field produces a deformation (magnetostriction strain or direct effect) and the application of a stress induces a 
magnetization of the material (inverse effect). It may be noted that, in the direct effect the magnetic field causes elastic deformation or variation of the Young's modulus, and in the inverse effect the mechanical stress modifies the magnetic properties: the magnetization curve (function) or the hysteresis loop. These two effects have been found separately. Joule discovered the direct effect in 1842. Two decades later, Villari exposed in 1864 that the stresses have an impact on the magnetization that do the sign of the deformation of the material govern by magnetostriction. Posterior, it was acknowledged that these two occurrences link to the same thermodynamic relation. It is worth notable that, it is recalcitrant to characterize magnetostriction-strains from measurements. This is not only for the reason that their amplitude is insignificant, but also for the reason that they strongly depend on texture, material constitution, and the applied stresses. The measured deformations outcome from unattached processes: pure magnetostriction strains and elastic deformations due to magnetic forces.

We can use the benefit of magnetostrictive materials properties for sensor and actuator applications. In addition, the magnetostrictive property besides being beneficial in such cases, for example, in ultrasonic applications, it could also present some undesirable effects in other applications. For example, the generation of acoustic noise in electromagnetic power systems (transformers, electric machines, etc.).

In the case of material intrinsic interactions, one may use different means for the modelling of the magneto elastic effects, depending on the preferred scale level of description. Such a choice depends on the need for investigation in terms of analysis, design or optimization. This may concern more precisely materials that magneto elastic intrinsic interaction corresponds to their self-functioning. For example, the quest for materials styled to specific applications e.g. transducers, calls for the use of well along constitutive models, accounting for coupled magneto-mechanical phenomena involved in such applications. One can consider more specifically typified the magneto-mechanical coupling in this case, by the stimulus of stress on the magnetic susceptibility (supporting the effective consequence of stress on the functioning of such devices) and by the magnetostriction (exploited in magnetostrictive transducers).

On the macroscopic scale, the two phenomena of the direct and inverse effects of magnetostriction, mentioned in the last section illustrate the coupling between the elastic and magnetic behaviors of ferromagnetic materials. The complexity of the nonlinear relationships of these phenomena is such that, it seems difficult to propose realistic macroscopic behavioral equations to model the coupled magneto-elastic behavior of magnetic materials. In other words, magnetostriction and magnetization are macroscopic manifestations of the complex structure of the magnetic domains that modified by the applied mechanical and magnetic loads.

In microscopic methodologies, we can define the structure at the atomic level and the materialization of magnetic domains may be the result of atomic interactions. The outcomes sent by such representations are intimate to physical observations, but they call for expensive computations whose extension is problematic up to a macroscopic scale. 
On the other hand, for macroscopic approaches, we can study the material as a continuum and thus we can define its condition by some evaluable and internal variables such as magnetization, magnetostriction strain and supposedly irreversible magnetization. One can use thermodynamic relations to derive complete constitutive relations. The calculation times may be low, but the structure of the magnetic domain is not involved. It is therefore, as mentioned before, difficult to describe with such one-scale model the couplings between the different variables and their evolution as well as phenomena occurring for complex loadings.

Different solutions accounting for both microscopic and macroscopic aspects based on specific assumptions and approximations permit to obtain material constitutive models, accounting for coupled magneto-mechanical phenomena; (see for example, Besbes et al. 2001, Buiron et al. 1999, Daniel and Galopin 2008).

A joint analysis of the magnetic and mechanical phenomena taking into account the magnetostrictive property requiring the use of a strongly coupled model is given in Besbes et al. (2001). This work considers the equations that govern the magneto-elastic phenomena associated with their boundary conditions and their laws of behavior. It presents the different forms of energy in a magnetostrictive material. An investigation of three cases of constitutive laws of behavior: the linear case, the nonlinear magnetic with linear elastic case, and the all-nonlinear case, is carried out. The energy functional corresponding to each case is developed. By minimizing the total functional energy, a local distribution of variables model of the problem in terms of magnetic vector potential and displacement is obtained. An example studied in this article illustrates the interaction phenomena between magnetic and elastic properties. This is clearly demonstrated through the modification of the distribution of the force and the deformation with respect to the situation without taking into account the magnetostrictive force.

In a kind of micro-macro or multiscale methodologies, Buiron et al. (1999) propose to use homogenization techniques to realize the macroscopic behavior of single crystals and polycrystals from a statistical picture of the magnetic domain structure. So, one can determine global values of magnetostriction strains. Therefore, the macroscopic couplings naturally get up from the expression of the free energy expressed at the level of the magnetic domains. Computation are longer than for macroscopic models, but more information can be determined from the microscopic scale (domain structure, texture, anisotropy) and the modelling of couplings between phenomena is generally simplified because expressed at a lower scale. In an application of the multiscale approach for magnetostrictive behavior modeling, Daniel and Galopin (2008) present, based on a statistical energetic description of the domain microstructure evolution, an analysis in the case of anhysteretic behavior, of the case of Terfenol-D single crystals and polycrystals. 


\section{Conclusions}

This paper endeavors to examine the two concepts of observational and mathematical modeling assessments by highlighting their specific uses in different branches of scientific exploration. We discussed the nature of the research domains involving observation, mathematical modeling, or both concerns. We explore the circumstances of model approaches that reflect their intimacy with observed reality. We have shown that in the case of such a reality corresponding to a societal application, the model often needs a reinforcement to advance towards its objective. We study how to take into account, in the model governing a societal application, the different theoretical domains involved in the modeling of a given device for a particular environmental behavior. We discuss the choice of such a coupling strategy according to the degree of interdependence of the scientific domains involved.

The contribution provides examples of different applications of coupled models in the case of electrical engineering (electromagnetic systems), related to different societal applications. In this case, we illustrated the need to consider the different behaviors involved in electromagnetic systems: electrical, magnetic, mechanical and thermal. The associations of these behaviors in weak or strong couplings clearly illustrate the concept of the model revisited to get closer to the observable reality (Razek 2017).

As general conclusions, first for the self-sufficiency of observation and modeling we can settle that both, except for particular cases, are complementary and materialize an associated couple. The observation needs modeling to permit a necessary generalizing context for outspread research. The model needs observation to verify its validity and to reinforce its nature to meet the observed reality. As a second conclusion, the observable is real and the model needs revision to match the observable. We can do such revision by associating the other theories corresponding to phenomena involved in the societal application. The revised model results from coupling of such associated models. The degree of coupling depends on the interdependence of the involved phenomena and the concerned behavior laws. The more, interdependent are the phenomena and nonlinear are the behavior laws, stronger will be the coupling (simultaneous solution of equations). In the contrary case, we need weaker coupling (direct or separate solution of equations). The third point concerns the developer and the users of the revised models. Developers are supposed to be engaged in skillful teams in applied mathematics, numerical analysis, computer science and the scientific fields relate to the developed tool; they are expected to work closely with the users. These users must be experts in the tool stipulations and implicated in the teams working in the heart of societal sceneries. 


\section{References}

Adkins DL, Boychuk J, Remple MS, Kleim JA (2006) Motor training induces experiencespecific patterns of plasticity across motor cortex and spinal cord. Journal of Applied Physiology 101(6): 1776-1782.

Amalric M, Dehaene S (2017) Cortical circuits for mathematical knowledge- Evidence for a major subdivision within the brain's semantic networks. Philosophical Transactions of the Royal Society B - Biological Sciences 373(1740): 20160515.

Ancelle B, Coulomb J, Morel B, Belbel E (1980) Implementation of a computer aided design system for electromagnet in an industrial environment. IEEE Transactions on Magnetics 16(5): 806-808.

Besbes M, Ren Z, Razek A (2001) A generalized finite element model of magnetostriction phenomena. IEEE Transactions on Magnetics 37(5): 3324-3328.

Buiron N, Hirsinger L, Billardon R (1999) A multiscale model for magneto-elastic couplings. Journal Physics 9(PR9): 187-196.

Daniel L, Galopin N (2008) A constitutive law for magnetostrictive materials and its application to Terfenol-D single and polycrystals. The European Physical Journal Applied Physics 42(2): 153-159.

Ferrari PF and Rizzolatti G (2014): Mirror neuron research - the past and the future. Philosophical Transactions of the Royal Society of London. Series B, Biological Sciences 369(1644): 20130169.

Knill DC, Pouget A (2004) The Bayesian brain: the role of uncertainty in neural coding and computation. Trends in Neurosciences 27(12): 712-719.

Konrad A (1981) The numerical solution of steady-state skin effect problems - an integrodifferential approach. IEEE Transactions on Magnetics 17(1): 1148-1152.

Lévi-Strauss C (1958) Structural anthropology. Paris.

Merleau-Ponty M (1960) The eye and the spirit. Paris.

Nakata T, Takahashi, N (1986) Numerical analysis of transient magnetic field in capacitor-discharge impulse magnetizer. IEEE Transactions on Magnetics 22(5): 526-528.

Nielsen JB, Cohen LG (2008) The Olympic brain. Does corticospinal plasticity play a role in acquisition of skills required for high-performance sports? The Journal of Physiology 586(1): 65-70.

Pawlak AM, Nehl TW (1988) Transient finite element modelling of solenoid actuators: the coupled power electronics mechanic and magnetic field problem. IEEE Transactions on Magnetics 24(1): 270-273.

Piriou F, Razek A (1990) Numerical simulation of a nonconventional alternator connected to a rectifier. IEEE Transactions on Energy Conversion 5(3): 512-518.

Piriou F, Razek A (1993) Finite element analysis in electromagnetic systems accounting for electric circuits. IEEE Transactions on Magnetics 29(2): 1669-1675.

Pouget A, Beck JM, Ma WJ, Latham PE (2013) Probabilistic brains: knowns and unknowns. Nature Neuroscience 16(9): 1170-1178.

Razek A (2017) For contributions to coupled multiphysics modeling and design of electromagnetic systems. https://www.ieee.org/about/awards/bios/tesla-recipients. html, IEEE_Nikola_Tesla_Award-2017.pdf.

Ren Z, Razek A (1990) A coupled electromagnetic - mechanical model for thin conductive plate deflection analysis. IEEE Transactions on Magnetics 26(5): 16501652. 
Ren Z, Razek A (1994) A strong coupled model for analyzing dynamic behaviors of nonlinear electromechanical systems, IEEE Transactions on Magnetics 30(5): 32523255 .

Sekkak A, Pichon L, Razek A (1994) 3-D FEM magneto-thermal analysis in microwave ovens. IEEE Transactions on Magnetics 30(5): 3347-3350.

Sekkak A, Kanellopoulos VN, Pichon L, Razek A (1995) A thermal and electromagnetic analysis in biological objects using 3D finite elements and absorbing boundary conditions. IEEE Transactions on Magnetics 31(3): 1865-1868.

Shen D, Meunier G, Coulomb J, Sabonnadiere J (1985) Solution of magnetic field and electrical circuit combined problem. IEEE Transactions on Magnetics 21(6): 22882291. 